\title{
On Application of KMV Model in Credit Risk Management of China's Securities Companies
}

\author{
Zhiqiang Chen $^{1,2,3}$, Hongmei Zhang ${ }^{1,2}$ \\ ${ }^{1}$ Guizhou Institution for Technology Innovation and Entrepreneurship Investment, Guizhou University of Finance \\ and Economics, Guiyang Guizhou 550025, China \\ ${ }^{2}$ Guizhou Institution for Urban Economy and Development, Guizhou University of Finance and Economics, Guiyang \\ Guizhou 550025, China \\ ${ }^{3}$ School of Finance, Guizhou University of Finance and Economics, Guiyang Guizhou 550025, China
}

\section{KMV 模型在我国证券公司信用风险分析中的应用研究}

\author{
陈志强 $1,2,3$, 张红梅 ${ }^{1,2,3}$ \\ 1 贵州财经大学贵州科技创新创业投资研究院, 贵州贵阳 550025 , 中国 \\ 2 贵州财经大学贵州城镇经济与发展研究院, 贵州贵阳 550025, 中国 \\ 3 贵州财经大学金融学院, 贵州贵阳 550025 , 中国
}

\begin{abstract}
As China's market-oriented and international level gradually increased, financial institutions, particularly, the securities firm's has faced much more risks which has showing a diverse, volatile trend. Through quantitative analysis methods, used KMV model to analyzes the credit risk of domestic securities companies. The results of research have shown that KMV model can be more accurate identification the credit risk of the securities companies. As well, China's securities companies are facing relatively large credit risks. So, securities companies should focus on improving the credit quality of the debtor and accelerating the establishment of the compliance's data management system, therefore securities companies' credit risk can be better identified and be controlled.
\end{abstract}

Keywords: KMV model; Credit risk management; Securities companies; Distance to default.

\section{摘 要}

随着我国市场化、国际化程度的逐渐提高, 金融机 构特别是证券公司面临的风险也呈现出多样化、波 动剧烈化的趋势。本文通过定量分析的方法, 运用 KMV 模型对国内证券公司的信用风险进行了分析。 实证结果表明我国 KMV 模型可以较为准确的识别
我国证券公司的信用风险, 且我国证券公司所面临 的信用风险相对较大。因此, 证券公司应该注重提 高债务人的资信质量并且要加快建立违约数据管理 系统, 从而更好的对证券公司信用风险进行识别和 控制。

关键词: KMV 模型; 信用风险管理; 证券公司; 违约距离

1. 引言

自从 1987 年 9 月我国成立第一家证券公司即深圳特 区证券公司以来, 证券公司在我国取得了蓬勃的发 展, 资产规模不断扩大、证券公司数量迅速增多。 证券公司在我国金融活动, 企业融资、筹资上扮演 了越来越重要的作用。从最一般的证券经纪业务、 投资咨询业务, 逐渐发展到证券承销与保荐业务、 证券自营业务、资产管理业务以及风险投资等业务。 其金融服务功能在不断扩大和完善的同时, 面临的 风险也呈现多样化的趋势, 其面临信用风险的可能 性也在增大。这意味着证券公司的管理者必须要对 公司面临的各类风险进行识别、度量、决策实施和 风险控制及评估。

传统的信用风险分析主要是从定性的角度出发, 比较有代表性的是专家制度法, 该方法指的公司在 发放贷款时由经验丰富的信贷管理员根据贷款人的 各项品质决定, 主要有 “ $5 \mathrm{C}$ ”、“ $5 \mathrm{~W}$ 等具体的信用 
Risk Analysis and Crisis Response in Big Data Era (RAC-16)

分析方法。该类分析方法在早期有较大的影响力, 但是专家制度法存在着很强的主观意识, 不利于债 权人的健康发展。 $\operatorname{Altman}(1968)^{[1]}$ 提出了 Z 计分模 型来分析信贷机构面临的信用风险, 该方法从传统 的定性分析逐渐转到定量分析上。在对以往文献的 查阅中我们可以发现, 传统的分析存在着主观性太 强、实用性差等缺点, 这也一定程度造成了信用风 险监管失误等弊端。现代信用风险分析主要是采取 计量的方法建立模型, 用定量的方法分析交易主体 的具体风险, 在这些定量分析方法中代表性的是 KMV 公司提出的 KMV 模型、JP Morgan 银行提出的基 于风险价值的 var 模型。

由于 $V a R$ 方法要求有足够的历史数据, 研究结 论必须基于正常的市场环境下才能解释结果等假设
级, 进而评价其面临的信用风险。对于 KMV 模型 的基本思想可以用图 1 直观的描述。

图 1 中横轴表示时间 $\mathrm{T}$, 纵轴表示资产价值 $\mathrm{V}$, 资产价值与负债价值的差额即所有者权益。在 $\mathrm{T}$ 时 刻当资产大于到期债务时, 违约距离为正值, 此时 认为不存在信用风险, 相反则存在信用风险。同样, 在 $T$ 时刻违约概率若为小概率事件或者为可承受的 风险敞口时认为信用风险相对较小。

\section{2. $2 \mathrm{KMV}$ 模型的基本假设}

由 $\mathrm{KMV}$ 模型的基本思想可知, 该模型是建立在 Merton 模型和 Black-Scholes 期权定价模型的基础 之上的, 故必须满足期权定价模型的如下基本假设 条件 ${ }^{[2]}$ :

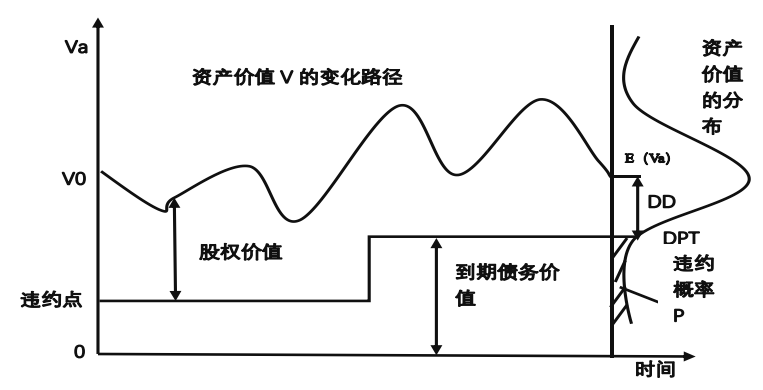

图 1. KMV 模型基本思想.

要求、传统信用风险管理方法主观性太强以及我国 证券公司受市场波动比较大等特点。出于以上几点 的考虑, 本文选用 KMV 模型对证券公司信用风险进 行分析。试图为我国证券公司分析其信用风险提供 新的解决思路, 从而更好的识别、分析、管理其信 用风险。

\section{KMV 模型理论简介}

\section{2. $1 \mathrm{KMV}$ 模型的基本思想}

KMV 模型是在资产定价模型和期权定价模型的基 础上建立的。KMV 模型使用了两个关系: 其一, 企业资产的市值波动程度和企业股票价格的变动程 度之间关系; 其二, 企业的股权市值与它的资产市 值之间的结构性关系。通过 KMV 模型利用这两个 关系可以求出企业资产的市值及其波动的程度。最 后, 算出相关变量的值, 信用监测模型便可以测算 出借款企业的预期违约频率(EDF)。并且根据违约 概率运用理论的违约概率测算表得出对应的风险等
首先, 公司的资产必须是可以自由交易的且服 从几何布朗运动:

$$
\mathrm{dV}=\mathrm{rVdt}+\sigma \mathrm{VdW}
$$

其中 $r_{A}$ 和 $\sigma_{A}$ 是无风险利率和资产的标准差, $W_{t}$ 是标准的维纳运动, 且服从期望为 0 , 方差为 $\mathrm{t}$ 的 正态分布;

其次, 完美市场假设。模型设定公司不支付股 利、股票不允许卖空、不存在税费支出, 公司在市 场中自由的筹资和投资且利率有较强的稳定性;

由于 $\mathrm{KMV}$ 模型的基本思想是通过资产价值和 违约距离来度量信用风险的大小, 因此还应该满足 以下几个假设:

首先, 注重考虑借款人的偿还能力而忽略借款 人偿还债务的意愿, 违约只发生在债务到期日, 公 司的股权价值和资产价值有如下关系:

$$
V_{e}=V_{a} N\left(d_{1}\right)-D e^{-r t} N\left(d_{2}\right)
$$

其中 $V_{e}$ 是公司的股权价值, $N\left(d_{1}\right) 、 N\left(d_{2}\right)$ 为 正态累计分布函数, 且 $d_{1}=\frac{\ln \left(\frac{V_{a}}{D}\right)+\left(r+\frac{\sigma_{a}^{2}}{2}\right) T}{\sigma_{a} \sqrt{T}}$ ； 
$d_{2}=d_{1}-\sigma_{a} \sqrt{T} ; \mathrm{N}(\mathrm{d})=\int_{-\infty}^{d} \frac{e^{-\frac{x^{2}}{2}}}{\sqrt{2 n}} d x 。$

根据股权价值对资产价值的弹性公式有:

$$
\mathrm{E}(\mathrm{VE}, \mathrm{VA})=\frac{\Delta \mathrm{Ve}}{V E} / \frac{\Delta \mathrm{Va}}{V A}=\frac{V_{A}}{V_{E}} \frac{\Delta \mathrm{Ve}}{\Delta \mathrm{Va}}
$$

由 (2) 式对 $V_{a}$ 求偏导并结合 (3) 式可以得出:

$\sigma_{e}=\frac{V_{a}}{V_{e}} N\left(d_{1}\right) \sigma_{a}$

其次, 债务人资本结构仅包含短期、长期借款、 所有者权益;

最后, 违约距离评价出的结果对于企业评价信 用风险是有用的 ${ }^{[3]}$ 。

\section{2. $3 \mathrm{KMV}$ 模型确定违约率的基本步骤}

对于上市公司来说, 股权价值和波动率可以通过二 级市场中的股票交易价格计算得出, 资产的价值以 及其波动情况要结合式 (1) 和式 (2) 解出。在得 出了股权价值和波动率后, 根据以下几步计算出 EDF (违约概率) ${ }^{[4]}$ 。

步骤 1 计算公司资产价值 $V_{a}$ 和其波动率 $\sigma_{a}$ 。

步骤 2 估计违约距离和违约点。根据以往经 验, 公司违约通常是在其资产价值等于公司 1 年期 及以下的短期负债加上长期负债的一半, 故把该值 假定为违约点。由图 1 中 KMV 的基本思想可知, 违 约距离指的是公司在 $\mathrm{T}$ 时刻资产价值与违约点的距 离 (DPT), 故可以定义为如下表达式:

$$
\mathrm{DD}=\frac{\ln \left(\frac{V_{a} a}{D P T}\right)+\left(r-\frac{\sigma_{a}^{2}}{2}\right) T}{\sigma_{a} \sqrt{T}}
$$

步骤 3 解得违约率 $\mathrm{EDF}$ 。目前对于违约率的计 算通常有两种思路: 即基于资产的价值分布的 EDF 即理论 $\mathrm{EDF}$ 和基于历史违约数据的经验 $\mathrm{EDF}$ 。考虑 到国内违约数据库尚未建立, 故在接下来的研究采 用的是理论 EDF。假设上市证券公司的资产价值服 从对数正态分布。故 EDF 可用如下公式表示:

$$
\mathrm{EDF}=1-\mathrm{N}(\mathrm{DD})
$$

经过以上的 3 个步骤的计算就可以得出 $\mathrm{EDF}$, 并且根据该值和理论违约 EDF 对证券公司的信用风 险作出相关的判断, 从而更好的识别证券公司在经 营中面临的信用风险。

\section{3. 数据来源和变量选取}

\section{1 数据来源及研究对象说明}

文中所使用的财务数据来源于 Wind 数据库。为了保 证研究的实效性, 选取的样本区间为 2016 年 1 月 4
日到 2016 年 3 月 31 日,计算的基准日是 2016 年 1 月 4 日。在选取样本区间时本文考虑到, 避开 2015 年股市大幅波动的情况, 目的是为了减少股市大幅 波动对研究结果的冲击。由上证指数在该样本区间 的 K 线图可知, 指数在 2638.3 点至 3678. 27 点之间 相对小幅的箱体运动, 此时股价更能反映公司的内 在价值, 这可以更加准确的研究证券公司面临的信 用风险。

考虑到国内对于证券公司信用风险的研究相对 贵乏, 以及各行各业所面临的信用风险差异性较大, 本文选取证券行业作为研究的对象。随着去年 6 月 份股市大跌, 国家出台限制相关证券业务的开展, 这给证券公司的经营产生了较大的冲击, 信用风险 也逐步暴露出来。基于这方面的考虑, 本文选取证 券公司作为研究对象。

此外，为了更好的分析 KMV 模型在我国上市公 司信用风险分析中的适用性的需要, 本文从我国的 A 股市场中选择了 6 只证券公司股票作为正常组, 其中 4 只为观察期内涨幅最大的股票, 2 只为观察 期跌幅最大的; 由于 ST 和*ST 股票存在退市风险、 信用风险较大, 故本文将这该类公司的股票作为对 比组, 选取了 A 股市场中 1 家 ST 板块的股票、5 家 $* \mathrm{ST}$ 为违约组, 其中选取方法和证券公司选取标准 一致。

\section{2 参数的确定}

股权价值 $\mathbf{E}: \mathrm{KMV}$ 模型通过股票的市值来衡量公司 的股权价值。虽然所研究的公司中有些公司股权是 全部流通的, 但是仍然有部分股票未全部流通, 为 了充分说明非流通股在我国上市公司价值反映中的 重要性,下文中的股权价值为 2016 年一季度各公司 的流通股市值加上非流通股的股数与每股净资产的 乘积的和来得出。

债务的价值 D（违约点 DPT）：根据 KMV 模型的 基本假设可知, 在计算中把公司的债务分为短期债 务和长期债务, 且长期债务按 $50 \%$ 的权重进行调整。 长期负债调整后的值加上短期负债的值即为公司 债务的价值 D。

无风险利率 $\mathbf{r}$ : 根据以往学者的研究经验, 文中选 用人民银行 2016 年一季度一年期定期存款利率作 为无风险利率 $r$ 。从万德数据库查询可知该值为 $1.5 \%$ 。

证券公司债务期限 T: 虽然证券公司的样本选取区 间为 2016 年 1 月 4 日到 2016 年 3 月 31 日,但数据 处理均为 1 年, 这样处理的目的是为了计算上的相 对简便。 
Risk Analysis and Crisis Response in Big Data Era (RAC-16)

证券公司股价波动率 : 对于该值, 本文选用样本区 间的收盘价作为当天的参考价, 并采用对数收益率 的方法计算公司股票的收益率, 最终运用统计学中 的标准差公式 $\sigma_{E}=\sqrt{\frac{\sum_{j=1}^{n}\left(u_{i}-\bar{u}\right)^{2}}{n-1}}$ 计算出股价收益波 动率。

\section{4. 基于 KMV 模型的证券公司信用风险实证分析}

\section{1 证券公司信用风险模型的运算}

第一步: 采用对数收益率及标准差公式计算出股价 日波动率, 在 Exce1 里运用迭代计算股票的股权价 值波动率;

第二步: 在 Excel 里计算样本公司违约点 DPT; 第三步: 根据公式 (2) 计算上市公司股权价值 $V_{e}$; 每股净资产和流通股收盘价格, 在里 Excel 计算样 本公司股权市场价值 $V_{E}$;

第四步: 首先, 在 Exce1 中准备好 $\sigma_{E} 、 r 、 V_{E} 、 \mathrm{D}$ 的 数据, 其次, 凭借 Matlab 编程的 Newton 迭代法, 在 Matlab 软件下运行以下的程序, 求解公式 (2)、 $\mathrm{d} 2$ 和 $\sigma_{\mathrm{A}}$ 以上非线性方程组。其中 $\mathrm{T}=1$, 其程序如下: M 文件:

clear all

clc

close all

$\mathrm{SigE}=$ xlsread('C: $\mathrm{U}$ sers $\backslash$ Desktop $\backslash K M V$ 模型一季度数 据','Sheet1','B2:B19');

$\mathrm{rf}=\mathrm{xlsread}(\mathrm{C} \mathrm{C}: \mid \mathrm{Users} \backslash$ Desktop $\backslash \mathrm{KMV}$ 模型一季度数据 ','Sheet1','c2:c19');

$\mathrm{VE}=\mathrm{x} 1$ sread $\left({ }^{\prime} \mathrm{C}: \backslash\right.$ Users $\backslash$ Desk top $\backslash \mathrm{KMV}$ 模型一季度 数据' , 'Sheet1' ,'D2:D19') ;

$\mathrm{DP}=\mathrm{x} 1$ sread (' C: $\backslash$ Users $\backslash$ Desktop $\backslash K M V$ 模型一季度

数据', 'Sheet1', 'E2:E19') ;

for $\mathrm{i}=1: 18$

$$
\mathrm{c} 1=\mathrm{DP}(\mathrm{i}) \text {; }
$$$$
\mathrm{c} 2=\mathrm{VE}(\mathrm{i}) \text {; }
$$

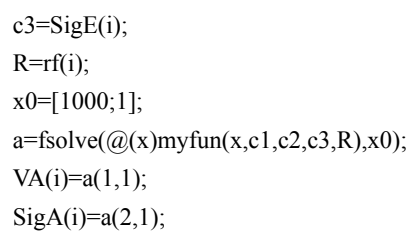

VA

\section{SigA}

上述程序中的文件即是方程组 $N(d) 、 d 1 、 d 2$ 和 $\sigma_{\mathrm{A}}$ 的 表达式, 迭代运算是通过 fsolve 函数实现的, 而 fsolve 函数需要最终输出的对应结果为变量 $V_{a}$ 和 $\sigma_{\mathrm{A}}$ 的两个初始值;

第五步: 根据第四步输出结果 $V_{a}$ 和 $\sigma_{\mathrm{A}}$ 以及公式 (5) 和 (6), 计算出样本公司的违约距离 DD 期违约概率 $\mathrm{EDF}$ 。

\section{2 模型的计算结果及分析}

基于 $\mathrm{KMV}$ 模型证券公司信用风险度量模型运用 Excel 迭代法、Matlab 的程序运算。最终的输出结 果为各证券公司资产价值和资产价值波动率, 输出 的值如下表 1 所示。

为了更加清晰的看出我国证券公司违约概率和 违约距离之间的关系。下面将用趋势图来表示上述 公司的信用情况，如图 2 所示。

由于我国证券公司违约数据库尚未建立，相关 数据缺乏，根据以上的结果并不能得出违约率对应 的信用等级情况。下文将从分别从违约距离、理论 违约概率的角度更加全面的分析证券公司的信用对 比情况。

\section{3 违约距离分析}

违约距离是衡量公司资产价值和债务价值的相对价 值。若一定期限内该值小于零, 则说明公司面临比 较严重的信用风险, 这时公司的股东或者债权人为

表 1 KMV 模型计算结果

\begin{tabular}{|l|l|l|l|l|l|l|l|l|l|}
\hline 公司 & VA & SigA & DD & EDF & 公司 & VA & SigA & DD & EDF \\
\hline 国元证券 & 68626 & 3.8 & 0.27 & $7.38 \%$ & ST 生化 & 237490 & 2.5 & 0.13 & $13.21 \%$ \\
\hline 太平洋 & 55343 & 4.8 & 0.31 & $5.50 \%$ & $*$ ST 皇台 & 5090 & 2.2 & 0.05 & $19.57 \%$ \\
\hline 东北证券 & 58118 & 3.1 & 0.44 & $2.19 \%$ & $*$ ST 钱江 & 4036 & 3.1 & 0.02 & $30.55 \%$ \\
\hline 招商证券 & 157125 & 6.8 & 0.17 & $8.43 \%$ & $*$ ST 南化 & 5906 & 1.2 & 0.07 & $26.97 \%$ \\
\hline 广发证券 & 196602 & 7.3 & 0.10 & $12.01 \%$ & $*$ ST 新集 & 49777 & 1.7 & 0.01 & $28.13 \%$ \\
\hline 中信证券 & 321507 & 6.4 & 0.14 & $11.85 \%$ & $*$ ST 商城 & 8385 & 3.1 & 0.02 & $27.42 \%$ \\
\hline
\end{tabular}

数据来源: 根据万德数据库, 经过 Exce1、Matlab 软件计算整理所得 
Risk Analysis and Crisis Response in Big Data Era (RAC-16)

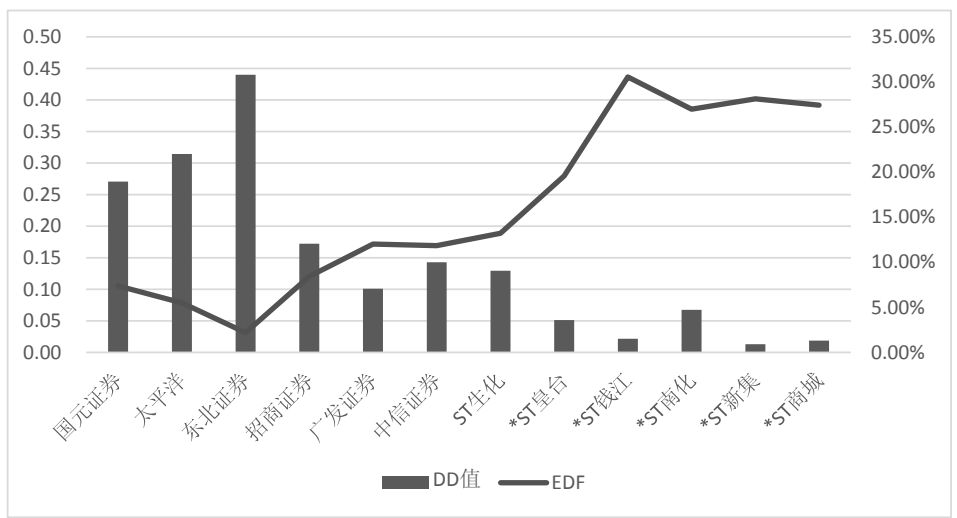

图 2 违约距离、违约概率组合图

数据来源: 根据万德数据库, 经过 Excel、Matlab 软件计算整理所得

了避免遭受损失, 可能会调整对公司未来的预期, 最终影响公司的日常经营。处于违约距离的重要性, 从违约距离 DD 值看, 上市证券公司、ST 公司、*ST 公司的违约距离的值都是相对较少的, 这一定程度 上说明我国 2016 年一季度公司整体经营情况较乐 观, 具体来看一季度证券公司的 DD 值最大, 说明 其面临的信用风险相对较小, 东北证券、太平洋、 国元证券的 DD 值相对较大, 说明这些公司的信用 风险管理相对较好, ST 公司、*ST 公司的 DD 值基 本保持在 0.1-0.5 之间, 这也说明这类公司的信用 风险比较大, 这符合这类公司信用情况较差的预期。

理论 EDF 是根据国外以往的研究经验, 把计算 出的 EDF 值和违约数据库对比, 以此估计出公司信 用等级。由于我国企业违约数据库尚未建立, 对比 的指标体系未形成。故本文借鉴以往的研究经验, 结合国外对信用等级划分的依据根据理论 EDF 对证 券公司及参考组公司的信用风险等级进行划分。

根据国际信用评级机构穆迪给出的 $\mathrm{EDF}$ 与信用 等级的关系可知, EDF 值位于 $1.9 \%$ 至 $4 \%$ 为 $\mathrm{BBB} /$ $\mathrm{BBB}^{-} ; 4 \%$ 至 $7.2 \%$ 为 $\mathrm{BBB}^{-} / \mathrm{BBB} ; 7.2 \%$ 至 $10.1 \%$ 为 $\mathrm{BB} / \mathrm{BB}^{-}, 10.1 \%$ 至 $14.3 \%$ 为 $\mathrm{BB}^{-} / \mathrm{B}^{+} ; 14.3 \%$ 至 $20.2 \%$ 为 $\mathrm{B}^{+} / \mathrm{B} ; 20.1 \%$ 至 $34.5 \%$ 为 $\mathrm{B} / \mathrm{B}^{-}$。对应该标准, 各公 司评级情况如表 2 所示。由该表可知, 上市证券公 司的信用等级在 BBB-/BBB 到 BB- $/ \mathrm{B}+$ 之间, 最优的 是国元证券，广发和招商证券信用等级相对较低; *ST 类公司的信用等级主要集中在 $\mathrm{B} / \mathrm{B}$-上, 这也说 明该类公司的信用风险较大。根据理论 EDF 的分析 可知, 相比之下, 证券类公司的信用风险要小于 ST 类、 $* \mathrm{ST}$ 类公司。

\begin{tabular}{|c|c|c|c|c|c|c|}
\hline $\begin{array}{l}\text { 公 } \\
\text { 司 }\end{array}$ & $\begin{array}{l}\text { 国元证 } \\
\text { 券 }\end{array}$ & 太平洋 & $\begin{array}{l}\text { 东 北 } \\
\text { 证券 }\end{array}$ & $\begin{array}{l}\text { 招 商 } \\
\text { 证券 }\end{array}$ & $\begin{array}{l}\text { 广发 } \\
\text { 证券 }\end{array}$ & $\begin{array}{l}\text { 中 信 } \\
\text { 证券 }\end{array}$ \\
\hline $\begin{array}{l}\text { 评 } \\
\text { 级 }\end{array}$ & $\mathrm{BB} / \mathrm{BB}$ & $\begin{array}{l}\mathrm{BBB} / \mathrm{BB} \\
\mathrm{B}\end{array}$ & $\begin{array}{l}\text { BBB/ } \\
\text { BBB }^{-}\end{array}$ & $\begin{array}{l}\mathrm{BB} / \mathrm{B} \\
\mathrm{B}^{-}\end{array}$ & $\begin{array}{l}\mathrm{BB}^{-} / \\
\mathrm{B}^{+}\end{array}$ & $\begin{array}{l}\mathrm{BB}^{-} / \\
\mathrm{B}^{+}\end{array}$ \\
\hline $\begin{array}{l}\text { 公 } \\
\text { 司 }\end{array}$ & $\begin{array}{ll}\text { ST 生 } \\
\text { 化 }\end{array}$ & *ST 皇台 & $\begin{array}{l}\text { *ST } \\
\text { 钱江 }\end{array}$ & $\begin{array}{l}* \text { *ST } \\
\text { 南化 }\end{array}$ & $\begin{array}{l}\text { *ST } \\
\text { 新集 }\end{array}$ & $\begin{array}{l}\text { *ST } \\
\text { 商城 }\end{array}$ \\
\hline $\begin{array}{l}\text { 评 } \\
\text { 级 }\end{array}$ & BB-/B ${ }^{+}$ & $\mathrm{B}^{+/} \mathrm{B}$ & $\mathrm{B} / \mathrm{B}^{-}$ & $\mathrm{B} / \mathrm{B}^{-}$ & $\mathrm{B} / \mathrm{B}^{-}$ & $\mathrm{B} / \mathrm{B}^{-}$ \\
\hline
\end{tabular}

注：依据穆迪评级标准

\section{5. 结束语}

综上所述, 本文运用 KMV 模型, 具体分析了 2016 年一季度我国证券公司的信用风险, 通过对高风险 的 ST 公司、*ST 公司的违约距离和预期违约概率的 分对比析可知, KMV 模型适用于我国证券公司的 信用风险分析。实证结果显示, 我国证券公司的信 用风险虽然小于 ST 公司、*ST 公司, 仍然存在信 用风险。特别是广发和中信证券, 其违约概率超过 了 $10 \%$, 这表明这两家公司的信用风险相对较大, 为了未来更好的经营发展, 这些公司必须要加强信 用风险管理。此外, 由于理论 EDF 分析采用的是美 国的经验数据, 该数据可能和我国的实际情况存在 差异, 建议我国加快违约数据库的建立, 从而使研 究结果更好的符合实际情况、以及指导我国证券公 司信用风险分析。 
Risk Analysis and Crisis Response in Big Data Era (RAC-16)

\section{参考文献}

[1]姜慧华. KMV 模型在我国商业银行信用风险度量 中的具体应用-—以工商银行和南京银行为例. 财经论坛. 2016 (05) : 224-227.

[2]陈晓晨. 基于 KMV 模型分析利率市场化冲击对商 业银行信用风险影. 商, Business. 37(199): 193-194.

[3]杨秀云, 蒋园园, 段珍珍. KMV 模型在我国商业银 行信用风险管理中的适用性分析及实证检验. 财 经理论与实践. 37 (199) : 34-40

[4] 马美芸. A 股中小上市公司 ST 风险预警一基于 KMV 模型的违约风险实证研究.上海:华东理工大 学, 2015.

[5]张涁. 基于模型的融资租赁公司信用风险度量研 究.上海:东华大学, 2014.

[6] 李薇. 基于 KMV 模型的上市公司信用风险度量研 究-A 股市场房地产行业的实证研究. 成都: 西南 财经大学, 2013.

[7]凌江怀, 刘燕媚. 基于 KMV 模型的中国商业银行 信用风险实证分析一一以 10 家上市商业银行为 例. 华南师范大学学报 (社会科学版). (05) : 142-148.

[8] Y. Yang, L. Li, Z.F. Zhou, W.Y. Fei. The Research on applicability of amended kmv model with different industries. Journal of Risk Analysis and Crisis Response, 2013,3(1):27-33.

[9]爱德华・阿特曼（Edward Altman）.财务比率、 判别分析及公司破产预测. 财务杂志. 1968,22.

[10]谢赤、赖琼琴、王纲金. 基于 JD-KMV 模型的 上市公司信用风险度量一一一个区域金融视角 下的实证研究. 经济地理. 2014, 34(6):137-141. 